\title{
Luther, Brenz und das Bilderverbot ${ }^{1}$
}

\author{
von TORSTEN KRANNICH
}

Das christliche Sakralbild unterlag von Anfang an dem Generalverdacht, dass es die Menschen vom wahren Glauben ablenken könnte, weil es Ehre oder gar Anbetung auf sich zieht, die Gott allein gebührt. Darum gab es in den christlichen Kirchen über alle Zeiten hinweg Auseinandersetzungen, ob man Gottes- und Heiligenbilder aufstellen darf ${ }^{2}$. Es waren vor allem zwei Phasen der Kirchengeschichte, in denen diese Frage besonders virulent wurde: Im achten und frühen neunten Jahrhundert wurde insbesondere in der Ostkirche darüber diskutiert, ob man durch die Darstellung Christi im Sakralbild seine Göttlichkeit einschränken würde. Die zweite Phase, in der man erbittert über den rechten Umgang mit Bildern diskutierte, war das Reformationsjahrhundert. Schon kurz nach Beginn der Reformation entzündete sich der Streit an der Frage, ob weiterhin Bilder in den Kirchen zugelassen sein sollten oder nicht.

Es waren vor allem zwei Personen, die sich für die Entfernung aller Bildwerke einsetzten: Andreas Bodenstein, genannt Karlstadt, (um 1480-1541) in Wittenberg und Huldrych Zwingli (1484-1531) in Zürich. Beide strebten eine Neuordnung des christlichen Lebens an, in deren Folge aber jede Form von Heiligenverehrung abgelehnt wurde. Ihnen ist zugleich ähnlich, dass sie durch eine strenge Auslegung der biblischen Texte eine wortwörtliche Bibelauslesung forderten. So kam es im Winter 1521/22 in Wittenberg und ab 1523 in Zürich immer wieder zu bilderstürmerischen Aktionen, bei denen die „Götzen“, d.h. die Heiligenbilder und die mit Bildern geschmückten Altäre aus den Kirchen entfernt wurden - teilweise verbrannt, teilweise auch ,nur“ eingelagert, um sie später zu verkaufen. Im Folgenden wird die Entwicklung der Bildertheologie bei Martin Luther (1483-1546) und Johannes Brenz (1499-1570) dargestellt. Während Martin Luther sich zur Bilderfrage schon zu einem Zeitpunkt äußerte, als es noch keinen Bildersturm gab, und er mithin seine Ideen primär als Konkretisierungen der Rechtfertigungslehre fassen konnte, entwickelte Johannes Brenz seine Bildertheologie auf dem Höhepunkt des reformatorischen Bildersturms und in der Auseinandersetzung mit diesem.

1 Für die anregenden Gespräche und Hinweise danke ich herzlich Prof. Dr. Franz Brendle (Tübingen), Prof. Dr. Hermann Ehmer (Stuttgart) und Peer Otte (Essingen).

2 Peter Hofmann: Bildtheologie. Position - Problem - Projekt (ikon. Bild + Theologie). Paderborn 2016, 17-128; Horst Schwebel: Die Kunst und das Christentum. Geschichte eines Konflikts. München 2002. 


\section{Martin Luthers Dekalogauslegungen von 1516 bis 1525}

Von Juni 1516 bis zur Fastenzeit 1517 hielt Martin Luther Predigten über die zehn Gebote. Die Predigten selbst sind leider nicht überliefert, aber er fasste seine Grundgedanken in Deutsch und Lateinisch zusammen. In beiden Texten wählt er denselben Aufbau: 1) Zitation der Gebote, 2) Übertretungen, 3) Erfüllung. ${ }^{3}$ Daneben veröffentlichte er 1518 eine lateinische Fassung seiner Predigten, von der wiederum 1520 eine deutsche Rückübersetzung herausgegeben wurde, vermutlich durch den Baseler Buchdrucker Sebastian Münster, der auch das Vorwort schrieb. ${ }^{4}$

Auffällig ist, dass schon in dieser ersten von Luther veröffentlichten Fassung der zehn Gebote das ursprünglich zweite Gebot des hebräischen Kanons - „Du sollst dir kein Bildnis noch irgendein Gleichnis machen, weder von dem, was oben im Himmel, noch von dem, was unten auf Erden, noch von dem, was im Wasser unter der Erde ist.“ (Exodus 20,4) - nicht erwähnt wird. Und auch in späteren Textausgaben des Dekalogs zitiert der Wittenberger Reformator dieses Gebot nicht, so z. B. in seiner kleinen Schrift Eyn kurez form der zeehen gepott (1520) ${ }^{5}$ oder im kleinen und großen Katechismus. ${ }^{6}$ Martin Luther bewegt sich mit seiner Auslassung des Bilderverbots ganz in der spätmittelalterlichen Tradition, in der das zweite Gebot oft auch nicht genannt wird. ${ }^{7}$

Auch wenn er keine eigene Predigt zum Bilderverbot hält, macht er schon 1516 in seiner ersten Predigt zum ersten Gebot deutlich, warum er auf eine eigenständige Auslegung zum Bilderverbot verzichtet: Er ordnet dieses Gebot dem ersten unter,

3 Martin Luther: Die tzehen gebothe gottes. In: Dr. Martin Luthers Werke. Kritische Gesamtausgabe. 120 Bde. 1889-2009 (künftig: WA), hier WA 1, S. 250-256; Ders.: Instructio pro confessione peccatorum (WA 1, S. 258-265).

4 Decem praecepta Wittenbergensis praedicta populo 1518 (WA 1, 398-521); Der zehen gebot gotes ain Schoene nutzlich Erklerung durch Doctor Martinum Luther Augustiner zu Wittenberg beschriben und gepredigt / gaistlichen und weltlichen dienende. Item ain Predig von den Siben todsünden D.M.L., 1520. Vgl. hierzu auch Johannes Geffcken: Der Bildercatechismus des funfzehnten Jahrhunderts und die catechetischen Hauptstücke in dieser Zeit bis auf Luther. I. Die zehn Gebote mit 12 Bildtafeln nach Cod. Heidelb. 438. Leipzig 1855, (10-16) 12; Michael Basse: Luthers frühe Dekalogpredigten in ihrer historischen und theologischen Bedeutung. In: Luther. Zeitschrift der Luther-Gesellschaft 78 (2007), S. 6-17, hier 9-13; Andreas Stegmann: Luthers Auffassung vom christlichen Leben (Beiträge zur historischen Theologie 175). Tübingen 2014, S. 258-285.

5 WA 7, 204-214.

6 Margarete Stirm: Die Bilderfrage in der Reformation (Quellen und Forschungen zur Reformationsgeschichte XLV). Gütersloh 1977, 17-21; Albrecht Peters: Kommentar zu Luthers Katechismen. Band 1: Die Zehn Gebote. Luthers Vorreden. Hg. von Gottfried Seebaß. Göttingen 1990, S. 86 f., 105-109, 106: „Mit den Memoriertexten des ausgehenden Mittelalters übergeht Luther das Bilderverbot".

7 Geffcken (wie Anm. 4), S. 58 f.; Veronika Thum: Die Zehn Gebote für die ungelehrten Leut'. Der Dekalog in der Graphik des späten Mittelalters und der frühen Neuzeit. München u. a. 2006, S. 18 f. Vgl. hierzu auch die Übersichten bei Stirm (wie Anm. 6), S. 235-240, die zusammenstellt, in welchen Katechismen bzw. spätmittelalterlichen Beichtbüchern das Bilderverbot zitiert wird und wo nicht. 
so wie alle Gebote immer aus dem ersten Gebot fließen. ${ }^{8}$ Luther führt Beispiele auf, in welcher Form sich Menschen von Gott abwenden und sich so ihre anderen Götter schaffen. Dazu gehört für ihn neben jeder Form von Wahrsagerei, Hexerei und Magie auch die Heiligenverehrung, da durch die Verehrung der Gläubigen die Heiligen selbst zu Abgöttern werden können. ${ }^{9}$ Luther führt dies an verschiedenen Beispielen aus, etwa wenn die Leute sich allein an den heiligen Antonius wenden, um sich vom Fieber heilen zu lassen, so als ob Gott nicht auch durch andere das Fieber nehmen könnte. ${ }^{10}$ Am Beispiel des heiligen Christophorus ${ }^{11}$ und seiner Bilder verschärft Luther seine Kritik noch mehr. Wenn Menschen meinten, wer am Morgen ein Christophorusbild gesehen habe, dem könne am Abend nichts geschehen: Woher sollte durch das Bild solch eine Kraft kommen? Und statt auf das Bild des Heiligen zu blicken, sollten die Menschen lieber auf das Kreuz sehen, denn dieses allein ist der wahre Christusträger. ${ }^{12}$

So kann Luther in der türkischen (d.h. muslimischen) Ablehnung aller Christusund Heiligenbilder sogar ein Werk Gottes sehen, weil diese durch die abgöttische Heiligenverehrung der Christen noch viel schlimmer bespien werden, als es den Türken möglich sei. ${ }^{13}$

Dass Luther das Bilderverbot als unmittelbaren Bestandteil des ersten Gebots versteht, zeigt er noch einmal explizit in einer Predigtreihe des Jahres 1525 über das Buch Exodus. Am 17. und 24. September 1525 predigte er über das 20. Kapitel. Nachdem durch Luther die zehn Gebote verlesen wurden, wobei er Ex 20,2-6 unter der Überschrift „,Das erste Gebot“ zusammenfasst, ${ }^{14}$ geht er in der folgenden Predigt überaus kritisch mit dem Dekalog um, da diese Texte, so Luther, nur dem Volk Israel gegeben wurden, weil es schon in Ex 20,2 heißt:

8 Haec praeceptum (sicut omnia alia) fluit ex primo (WA 1, 430,9), vgl. Basse (wie Anm. 4), S. 13; Peters (wie Anm. 6), S. 88. Dies spricht gegen Ehmers These, dass sich Luther erst aufgrund der Wittenberger Unruhen und dem von Karlstadt geplanten Bildersturm mit der Bilderfrage beschäftigte (Hermann Ehmer: Das Uracher Bildergespräch. In: BWKG 90 (1990), S. 67-91, hier 81).

9 [...] sanctos prope in idola sibi transformant (WA 1, S. 411,11-14).

10 WA 1, S. 412,8-15.

11 Spätmittelalterliche Christophorusreime finden sich etwa in den Erläuterungen zu den Schilderungen eines ehemaligen Kaplans, der um das Jahr 1531 eine ausführliche Beschreibung der Kirche St. Martin vorlegt, um zu zeigen, was durch die Reformation in Biberach an der Riß alles an sakralen und liturgischen Gegenständen aus der Kirche und ihren Kapellen verloren gegangen ist (vgl. Die religiösen und kirchlichen Zustände der ehemaligen Reichsstadt Biberach unmittelbar vor der Einführung der Reformation. Geschildert von einem Zeitgenossen. Hg. von A. Schilling. In: FDA 19 (1887), S. 1-191, hier 52 f. mit Anm. 2).

12 Cur non Crux Christi inspecta facit ridere inspectores, quae habet authoritatem scripturae? Nisi melior forte imago Christopheri quam Christi, cum crux Christi ipsa sit vere sola quidam Christopherus (WA 1, S. 413,28-30). Wenige Zeilen später spitzt der Übersetzer Sebastian Münster Luthers Text im Blick auf die Bilder noch einmal zu und lässt rhetorisch fragen: Wenn Christopherus zu seinen auch ainen solchen guten hailigen und beschirmer het gehabt, das er ain gantzen tag sicher gewesen wär / wenn er sein bild het angesehen / das in got net het geschlagen [...] (Münster, d iii). Vgl. zur Kritik an den Christophorusbildern auch Stirm (wie Anm. 6), S. 32.

13 WA 1, S. 421,21-27.

14 WA 16, S. 422,20-30. 
„Der dich aus Ägyptenland geführt hat" ${ }^{15}$ Mithin lehnt er eine strikte Befolgung des Dekalogs ab, dies tun nur Rottengeister ${ }^{16}$ und Schwärmer, ${ }^{17}$ die nicht erkennen können, was Gott zu einzelnen gesagt hat und was allen Menschen gilt. ${ }^{18}$ Denn eine wortwörtliche Umsetzung des gesamten Dekalogs bindet den Menschen nur, doch durch Christus gibt es Gnade. ${ }^{19}$ So wie im Alten Testament die Beschneidung das Zeichen des Bundes zwischen Gott und seinem Volk ist, so gilt das Bilderverbot auch allein den Juden. Doch für die Heiden(christen) gilt beides nicht mehr, so Luther unter Verweis auf Paulus (vgl. Röm 4,11):

Also sag ich hie, das das bildstuermen und umbreissen der goetzen nicht mag erzwungen werden aus diesem text, denn er ist den Jueden allein gesagt und nicht uns. Weise mir einen text, damit mir Gott verpoten hat die bilder. ${ }^{20}$

Derselben Argumentationslinie folgt seine Auslegung zum Bilderverbot. Das Bilderverbot ist von Gott allein an das Volk Israel gerichtet, so wie er auch allein Noah befahl, die Arche zu bauen (vgl. Gen 6,13-16) oder wie Christus nur Petrus den Auftrag gab, die Angel auszuwerfen, um im Maul des Fisches den Tempelgroschen zu finden (vgl. Mt 17,24-27). ${ }^{21}$

Nachdem Luther so die Forderung zurückgewiesen hat, dass die Bilder zerstört werden müssen, weil es im Dekalog steht, setzt er sich dennoch inhaltlich mit der Bilderverehrung auseinander. Wie schon in seiner frühen Predigt von 1516 ordnet er das Bilderverbot unter das erste Gebot, denn was hyndert mich ein bild, wenn mein hertz nicht daran hanget? ${ }^{22}$ In einem zweiten Schritt argumentiert Luther, dass sich auch ein geistliches Bild nicht von einem weltlichen Gemälde oder Schnitzwerk unterscheide. Es ist allein der Mensch, der dem Heiligenbild durch seine Verehrung einen höheren Stellenwert zuerkennt, wodurch er aber nicht nur sein Geld verliert, sondern auch das Heil: Denn wir haben bisher unser Frawen, Sant Annen, Crucifix und der gleichen bilder gemacht und die meynung darzu gehabt, daß besser weren denn ander holtz und steyn [...] Da brachten sie uns nicht allein umbs gelt, sondern auch umb die seel. ${ }^{23}$

\footnotetext{
15 WA 16, S. 424,14f.

16 WA 16, S. 430,23 .

17 WA 16, S. 431,8 . 16 , S. 429,30-33).

20 WA 16, S. 439,19-22.

21 WA 16, S. 438,13-21.

22 WA 16, S. $440,10 \mathrm{f}$.

23 WA 16, S. 440,14-19.
}

18 Luther nutzt als Beispiel für die Unterscheidung die Schöpfungsaussagen aus Genesis 1. Wenn es dort etwa heißt: „Die Fische sollen im Meer schwimmen und die Vögel in der Luft fliegen“, so ist das zwar auch Gottes Wort, aber niemand kommt auf die Idee, dass damit die Menschen gemeint seien (wilt du daruemb ein fisch werden und ym wasser wonen ... wilt daruemb zur sonnen, mond und sternen werden? [WA 16, S. 430,28-30]).

19 Wir haben ein ander werck und einen andern lerer, der nicht wie Moses zwinget und schrecket, sondern gnade anbeut, troestet, gibt und hilfft und erredtet, nemlich Jhesum Christum (WA 
Für Luther befindet sich der Bildersturm strukturell auf derselben Ebene wie die Bilderverehrung. Beide betreiben Abgötterei, weil sie hier wie dort meinen, für Gott ein gutes Werk zu vollbringen. Ikonolatrie und Ikonoklasmus sind mithin nur zwei Seiten einer Medaille. Darum brauchen die Menschen Unterweisung, um zu erkennen, dass sie allein von Gottes Gnade leben: man mus das volck mit dem wort dahyn bringen, das sie kein zuversicht haben zum bildern [...] Wo aber das volck unterweisset wuerde, das fuer Gott nichts helffe denn sein gnade und barmhertzickeit, so wuerden die bilden von yhn selber wol fallen und yhn verachtung komen $[\ldots]^{24}$.

Und so kommt er abschließend zu dem Urteil, dass das Bilderverbot lediglich dazu dient, sich nicht von den äußerlichen Dingen abhängig zu machen, sondern allein auf Gott zu vertrauen. ${ }^{25}$

\section{Johannes Brenz und die Bilder}

\subsection{Brenz als Befürworter eines strikten Bilderverbots. Die „Underrichtung der zwispaltigen Artickel" von 1524}

Wohl im Spätherbst/Winter $1524^{26}$ verfasste Johannes Brenz seine Schrift Underrichtung der zwispaltigen artickel cristenlichs glaubens, ${ }^{27}$ in der er der katholischen Lehre die neuen reformatorischen Positionen gegenüber stellt. In dieser Schrift vertritt Brenz eine überaus bilderkritische Position. Dabei beruft er sich zu Beginn seiner Argumentation auf einen kurzen Abschnitt aus der Schrift Divinae institutiones (Göttliche Unterweisungen) des spätantiken Apologeten Firmianus Lactantius (um 250-325 n.Chr.). Er zitiert Laktanz, ${ }^{28}$ wonach schon

24 WA 16, S. 440,19-29.

25 WA 16, S. 443,25-34. Vgl. auch in Georg Rörers Nachschrift die kurze Formel: Ergo illae imagines sunt prohibitae quae sunt contra verstand, quem vult deus. (S. 443,10 f.). Nach Gudrun Litz: Die reformatorische Bilderfrage in den schwäbischen Reichsstädten (Spätmittelalter und Reformation NR 35). Tübingen 2007, S. 23 f., sind für Luther Predigt und Seelsorge die Orte, in denen man dem Missbrauch der Bilder entgegentreten kann.

26 Vgl. zur Datierung das Einleitungskapitel zum Text, S. 56 f.

27 Eine ausführliche Darstellung der Schrift bei Martin Brecht: Die frühe Theologie des Johannes Brenz (Beiträge zur historischen Theologie 36). Tübingen 1966 (zugl. Habil. Tübingen 1964), S. 36-56; Friederike Nüssel: Allein aus Glauben: zur Entwicklung der Rechtfertigungslehre in der konkordistischen und frühen nachkonkordistischen Theologie (Forschungen zur systematischen und ökumenischen Theologie 95). Göttingen 2000, (zugl. Habil. München 1998), S. 61-70; zur Datierung Christian Peters: Brenz und das reformatorische Bekenntnis. In: BWKG 100 (2000), S. 9-28, hier 12.

28 Zur Kritik von Cicero an der Bilderverehrung, dem Laktanz folgte, vgl. Rudolph Kremer: Glaube und Aberglaube. Wie aus Religion und Superstition ein Gegensatz wurde. Marburg 2016, S. 52 60, hier 57. Im Rahmen des Bilderstreits des 16. Jahrhunderts wurde Laktanz auch von Heinrich Bullinger als Gewährsmann herangezogen, vgl. hierzu S.-P. Bergjan: Heinrich Bullinger, patristische Quellen und historische Arbeit in der Behandlung der Bilderfrage. In: Emidio Campi / Peter Opitz (Hg.): Heinrich Bullinger. life - thought - influence. Zürich 2004, August 25-29, 2004; Inter- 
seit alten Zeiten Menschen Bilder ihrer herausgehobenen Könige verfertigt hätten, um sich nach dem Tod derselben an sie erinnern zu können und einen Trost zu haben. Doch aus dem Trost wurde immer mehr Verehrung und am Schluss entstand daraus Aberglaube, denn die Römer haben die Verstorbenen divinisiert und so in den Himmel gesetzt. ${ }^{29}$

Unmittelbar an diesen Kirchenväterbeweis schließt Johannes Brenz nun die Zitation des Bilderverbots nach Ex 20,4f. bzw. Dtn 4,16-19 an und fasst dieses zusammen: Dis gepott verstet man nit allain von den gepildnussen der abgotter, sonder von allerley pildnussen Gottes und der heyligen, dan es stet also: Mach dir kain pildnuß deren ding, so im hymell sein. So ist ie Got und seine heyligen im hymell, darumb darff man ire pildnus nit machen. ${ }^{30} \mathrm{Um}$ auch die geringsten Zweifel an dem strikten Bilderverbot auszuräumen, betont Brenz, dass nicht nur die Verehrung der Bilder verboten sei, sondern schon deren Anfertigung: Es sein aber drey stuck furderlich an den bildnissen verbotten, nemlich machen, anbetten und eren oder dienen. ${ }^{31}$

In einem dritten Argumentationsstrang setzt sich Brenz mit der These auseinander, dass die Bilder als laicorum litteratura dienen..$^{32}$ Aber nach Dtn 6,6-9 sind es nicht die Bilder, sondern es ist allein nützlich, Gottes Wort zu lehren. ${ }^{33}$ Ebenso sind nicht die aus Stein oder Holz hergestellten Bilder Ebenbilder Gottes, sondern es ist der Mensch selbst (vgl. Gen 1,26f.). Für den gehöre es sich bekanntlich nicht, so Brenz, dass er auf einem Altar stehe. ${ }^{34}$ Selbst wenn die Men-

national Congress Heinrich Bullinger (1504-1575) (Züricher Beiträge zur Reformationsgeschichte 24). Zürich 2007, S. 389-406, hier 392 f.

29 Underrichtung der zwispaltigen artickel cristenlichs glaubens. In: Johannes Brenz, Frühschriften Teil 1. Hg.. von Martin Brecht u.a. Tübingen 1970, S. 55-111, hier S. 89a (94,2-17). Johannes Brenz zitiert hier Lactantius: Divinae institutiones I 15,3-7 (Corpus Scriptorum ecclesiasticorum Latinorum [CSEL] 19. Ed. Samuel Brandt und Georg Laubmann. Wien, Prag. Leipzig 1890, S. 55,20-56, 10; die Seitenangabe in der Brenz-Textausgabe lautet fälschlich „57f.“). Laktanz wiederum gibt zwei Stellen aus Ciceros Schrift „De natura deorum“ (II 24,62 und III 19,50) wieder und benennt Cicero auch als seinen Gewährsmann. Johannes Brenz unterschlägt in seiner Zitation den Hinweis auf Cicero, wie er im Zitat auch alle Namen der antiken Könige, die bei Cicero und Laktanz erwähnt werden (z.B. Heracles und Aesculap), auslässt (CSEL 19, SW. 56,3 f.). Leider verweisen die Herausgeber der Underrichtung in ihrem Apparat auch nicht auf diese pagane Quelle.

30 Underrichtung (wie Anm. 29), S. 89b (94,25-29).

31 Ebd., S. 90a (95,3-5).

32 Es handelt sich dabei um einen Gedanken, den erstmals Gregor der Große (um 540-604 n.Chr.) äußerte: „Was denen, die lesen können, die Bibel, das gewährt den Laien das Bild beim Anschauen, die als Unwissende in ihm sehen, was sie befolgen sollen, in ihm lesen, obwohl sie die Buchstaben nicht kennen, weshalb denn vorzüglich für das Volk das Bild als Lektion dient“" (Gregor d. Große, ep. XI 10 an Bischof Serenus von Marseille [MGH Epistolae II, S. 270,14-16]). Vgl. hierzu auch Walther von Loewenich: Bilder V/2. Im Westen, Theologische Realenzyklopädie in $27 \mathrm{Bdn}$. Hg. von Gerhard Krause und Gerhard Müller. Bd. VI, Berlin/New York 1980, S. 540-546, hier $543 \mathrm{f}$.

33 Underrichtung (wie Anm. 29), S. 90b: Es sprechen auch viell, die pildnuß seyen der layen pucher und rhaitzen zur andacht. Antwort: Der Her hat sein wort furgestelt, am selbigen sol man leren und nit ab den bildnussen, Deut 6 (6-9): Diese wort [...] (S. 95,20-23).

34 Ebd., S. 91a (95,29-96,2). 
schen Bilder nur zur Erinnerung und zur Mahnung aufstellen würden, käme es doch ganz schnell zum Missbrauch der Bilder, der dann zur Abgötterei würde. Brenz geht sogar soweit zu schreiben: Got hast die pilder, die mentschen aber zieren sie mit gold und berlen. ${ }^{35}$

Abschließend setzt sich Brenz mit der ehernen Schlange (Num 21,9) als alttestamentlichem Kultbild auseinander. Dabei handele es sich um eine besondere Beauftragung Gottes an Mose, die schon auf Christus verweisen soll (vgl. Joh 3,14), wie auch der von Mose für die Stiftshütte errichtete Thron Gottes auf Christus hinweise (vgl. Hebr 4,16). ${ }^{36}$ Brenz deutet hier noch einen Gedanken an, den er 1527 in seinem Johanneskommentar und 1528 in den Fragstucken weiter ausführen wird, dass nämlich durch die Inkarnation Christi alle Bilder überflüssig werden. ${ }^{37}$

Wir finden in dieser Frühschrift mithin eine Argumentation, die sich eng an Zwinglis Position anlehnt. Die Bilderverehrung ist für Zwingli eine erweiterte Fassung der Heiligenverehrung und wie diese auch abzulehnen. Denn für beides gibt es keine Belege in der Bibel, sondern sogar ausdrückliche Verbote. Einen wie auch immer gearteten pädagogischen Mehrwert kann Zwingli in Bildern nicht finden, vielmehr dienen die Bilder schon in ihrer künstlerischen Gestaltung vor allem dem moralischen Abfall der Menschen. ${ }^{38}$

Leider ist nur in geringem Maße ersichtlich, welche Rezeption diese frühe Schrift von Johannes Brenz erfahren hat. Da von ihr nur zwei Handschriften existieren und sie auch erstmals in Martin Brechts Habilitationsschrift von 1966 bzw. in der textkritischen Ausgabe der Frühschriften von 1970 einer größeren Öffentlichkeit bekannt gemacht wurde, blieb ihre unmittelbare Wirkung doch sehr überschaubar. So erklärt sich vielleicht auch, warum die hier vorgenommene explizite Ablehnung aller geistlichen Bilder in den Kontroversen der Zeit keine weitere Aufnahme fand. ${ }^{39}$

35 Ebd., S. $91 b(96,14-16)$.

36 Ebd., S. 92a (96,20-26). In der Ausgabe von Brecht et al. wird hier fälschlich als Bibelstelle „Rhom 3 [15]“" angegeben.

37 Ebd., S. 92a: Darumb auch, dweil Christus, die wahrhait, kumen ist, so sein aus ordnung Gottes der eusserlich gnadenthron und geflugelte pildnus zugrund gegangen. (96,26-29).

$38 \mathrm{Vgl}$. Huldrych Zwingli: Auslegung und Begründung der Thesen oder Artikel 1523. In: Huldrych Zwingli Schriften II, Zürich 1995, S. 254 ff. Vgl. Hans-Dietrich Altendorf: Zwinglis Stellung zum Bild und Tradition christlicher Bildfeindschaft. In: Ders. / Peter Jezler (Hg.): Bilderstreit. Kulturwandel in Zwinglis Reformation. Zürich 1984, S. 11-18, hier 13-15; Stirm (wie Anm. 6), S. 130153.

39 Anders: Peters (wie Anm. 27), S. 11 f., der von einer bedeutsamen Rezeption des Textes spricht, dafür aber lediglich auf eine (!) wörtliche Wiedergabe im Rahmen einer Heilbronner Verteidigungsschrift der Confessio Augustana verweisen kann. Berndt Hamm: Lazarus Spengler (1479-1534). Der Nürnberger Ratsschreiber im Spannungsfeld von Humanismus und Reformation, Politik und Glaube (Spätmittelalter und Reformation 25). Tübingen 2004, S. 289 mit Anm. 22, folgt der Argumentation von Peters. 


\subsection{Auslegungen zu Joh 1,18 „Niemand hat Gott je gesehen“ 1527 und 1528}

Im Jahr 1527 veröffentlicht Johannes Brenz in erster Auflage einen Kommentar zum Johannesevangelium. Er legt darin den Vers aus dem Johannesprolog (Joh 1,18) „Niemand hat Gott je gesehen; der Eingeborene, der Gott ist und in des Vaters Schoß ist, der hat ihn uns verkündigt." mit Hilfe von Ex 33,12-23 (Mose begehrt Gott zu schauen) aus. Wer heute Gott schauen will, so Brenz, muss Christus schauen, denn „,niemand kann Gott sehen, wenn er nicht sein Angesicht und sein Bild betrachtet." ${ }^{\text {" } 0}$ In Anlehnung an das hohepriesterliche Gebet (Joh 17,1-26) kann Brenz nun von der Einheit von Vater und Sohn sprechen, die erst durch die menschliche Natur Jesu für die Menschen sichtbar wurde, weswegen Mose einst noch nicht Gottes Angesicht sehen konnte, ${ }^{41}$ was uns aber heute möglich sei. Auch wenn es naheläge, sich von dieser Argumentation her zur Bilderfrage zu äußern und z.B. noch einmal die Bilderkritik aus der „Underrichtung“ zu wiederholen, unterlässt Brenz es.

Ohne weiter im Blick auf die Bilderfrage einzugehen, bietet Brenz im Kommentar in seinen Ausführungen zu Joh 8,27 (,Meinen Frieden gebe ich euch“) die auch von Luther bekannte Kritik an den angeblich einen Tag lang schutzverheißenden Christophorusbildern. ${ }^{42}$ Für Brenz sind es nicht die Bilder, sondern allein das Wort Gottes, welches Hilfe bringt. ${ }^{43}$

Ein Jahr später nimmt Brenz in den von ihm für den Druck nicht autorisierten Fragstucken die Rede von der Nichtsichtbarkeit Gottes nach Joh 1,18 noch einmal auf. Doch nun bekommt die Auslegung des Bibelverses eine bilderkritische Zuspitzung. Die Schrift ist so etwas wie ein Probelauf seines späteren Katechismus, unterscheidet sich aber deutlich von der Fassung von 1535.44

In den letzten beiden Fragen zum ersten Artikel des apostolischen Glaubensbekenntnisses heißt es im Catechismus maior, der an Erwachsene gerichtet ist. ${ }^{45}$

40 In D. IOHANNIS EVANGELION, IOANNIS Brentij Exegesis, in qua, praeterquam quod fere omnes qui unquam in Iohannem scripserunt, facile antecellit, Eucharistias etiam negocium cum pie, tum faeliciter exequitur. Cum Privilegio Imperiali, Haganae, per Ioa, Sece M.D. XXVII, (künftig zitiert comm. in Joh), S. 16a: Ita Deus nemo videre potest, nisi faciem et imaginem eius contempletur. Die Werke von Brenz werden nach dem Werkverzeichnis der Bibliographia Brentiana. Bearb. von W. Köhler. Berlin 1904 mit der in der Brenz-Forschung gebräuchlichen Zählung versehen, hier: Köhler 22.

41 Ebd., S. 17a: faciem aut mea uidere non poteris: de humanitate Christi [...] faciem eius non uidit, quia nondum erat Verbum incarnatus.

42 Ebd., S. 261a. Vgl. hierzu auch seine Polemik zur Verehrung der 14 Nothelfer in seiner Predigt Ein sermon von den heyligen / gebredigt zu Schwebisch hall durch Mayster / hansen brentz an sant iacobs tag 1.5.23, S. 1a-3a, abgedruckt in Brenz Werke Frühschriften (wie Anm. 29), Bd. 1, S. $5,1-8,16$.

43 Comm. in Ioh (wie Anm. 40), S. 261a: crede certo, Dei Verbo tibi bona optari.

44 Christoph Weismann: Brenz und seine Katechismen. In: BWKG 100 (2000), S. 123-132, hier $123 \mathrm{ff}$.

45 Christoph Weismann: Die Katechismen des Johannes Brenz 1. Die Entstehungs-, Text- und Wirkungsgeschichte (Spätmittelalter und Reformation 21). Berlin/New York 1990, S. 73-138, hier 108. 
Frag: Ist Got auch sichtbarlich? Antwort: Nayn

Frag: Woher kennest Du dann Gott? Antwort: Auß der Predig seins aingebornen Suns unsers herren Jesu Christi / wie geschrieben stehet / Gott hat niemandt gesehen / aber sein aingeborner Sun, der da ist in seins vatters ${ }^{46}$

Wir finden in dieser extrem verkürzten Aussage eine traditionelle ikonoklastische Argumentation gegen die Gottesdarstellung im Bild vor. ${ }^{47}$ Brenz rekurriert nicht auf das alttestamentliche Bilderverbot, sondern argumentiert wiederum streng von der Unsichtbarkeit Gottes unter Verweis auf Joh 1,18.48

Wir haben mithin innerhalb kurzer Zeit zwei divergierende Deutungen derselben Bibelstelle. Zum einen spricht Brenz in seinem Johanneskommentar davon, dass Gott dank der Inkarnation Jesu und dessen menschlicher Natur für uns sichtbar ist. Die sich daraus ergebende logische Folge seiner Abbildbarkeit denkt er jedoch nicht an. Zum anderen kann er aber auch dieselbe Bibelstelle nutzen, um die Nichtsichtbarkeit Gottes zu betonen.

Dass Brenz mit dieser konträren Auslegungstradition von Joh 1,18 zu seiner Zeit nicht allein stand, können wir aus anderen Äußerungen des 16. Jahrhunderts nachweisen. Genutzt wird Joh 1,18 im ikonoklastischen Sinne in einem Gedicht eines anonymen Bildkritikers, der seinen Verteidiger des Schweizer Bildersturms sagen lässt: Wenn man einen wolt Figuriren / Und wie ein Narren abpossiren [i. S. v. abbilden], Würd er das Bild nicht balt zubrechen / Und sich stracks an dem Maler rechen? / Wer hat nun Gott jemals gesehn, / Oder sich dürfen zu ihm nehn [nahen]. ${ }^{49}$

Im bilderfreundlichen Sinne findet sich die Stelle dagegen in einer frühen Zürcher Disputation aus dem Jahre 1523, an der u. a. Huldrych Zwingli teilgenommen hat. Während Leo Jud (1482-1542) wie Zwingli eine strikte Abschaffung

46 Fragstuck des Christlichen glaubens für die Jugendt zu Schwebisch Hall J.B.E.H. (Köhler 30) - (Im Folgenden: Fragstuck), hier Fragstuck zum ersten Artikel, VIv-VIIr (auch abgedruckt in: Die Evangelischen Kirchenordnungen des XVI. Jahrhunderts. Begründet von Emil Sehling. Bd. XVII/1. Baden-Württemberg 3, Südwestdeutsche Reichsstädte Teilbd. 1. Schwäbisch Hall, Heilbronn, Konstanz, Isny und Gengenbach. Tübingen 2007, Nr. 5, 84 [künftig: EKO]). In der überarbeiteten Fassung der Fragstuck von 1532 werden die Formulierungen in derselben Form beibehalten. Durch die Weglassung des Catechismus maior im Katechismus von 1535 findet sich die Aussage nicht mehr. Vgl. zu den beiden Fassungen Christoph Weismann: Die Katechismen des Johannes Brenz 2. Bibliographie 1528-201, mit einem Vorwort von Hermann Ehmer und einem Register von Christoph Brecht und Martin Brecht (Spätmittelalter und Reformation 22). Berlin/Boston 2016, S. 116-121. 47 Vgl. hierzu den Horos der ikonoklastischen Synode von Hiereia (Joannes Dominicus Mansi: Sacrorum conciliorum nora et amplissima collection. Vol. 13, Graz 1960, 252 A - 280 E). Siehe auch: Die ikonoklastische Synode von Hiereia 754. Einleitung, Text und Kommentar ihres Horos besorgt von Torsten Krannich u.a. (Studien und Texte zu Antike und Christentum 15). Tübingen 2002, S. 9-27.

48 Vgl. auch 1 Joh 4,12 (Niemand hat Gott jemals gesehen.).

49 Anonymus, Eiconoclasta. Dialogus oder Gespräch Von den Götzen 8, (zitiert nach: Jörg Jochen Berns: Von der Strittigkeit der Bilder. Texte des Deutschen Bilderstreits im 16. Jahrhundert. Bd. 2. Berlin/Boston 2014, Nr. 54, S. 1012). 
der Bilder forderte, ${ }^{50}$ erklärte sein Mitdisputant Heinrich Lüthy, der zu dieser Zeit in Zürich als zweiter Pfarrer am Münster wirkte: Ja, ich wey $\beta$ wol, der gottheit halb sol man jn nit abmalen. Es ist aber nit verboten, Christum, so ferr [fern] er ein mensch ist, abzebilden. Ich wey $\beta$ sust wol, das gott nie niemant gesehen hat, Epi. 1. Ioan. 4. [1 Joh 4,12]: ,Gott hat nieman gsehen. 'Deßglychen in der bschrybung sins Evangeliums capi. 1. [Joh 1,18], Item am 5. [Joh 5,37]: ,Gott hat nie yemant gesehen, und sin stimm hat niemant gehört.' Das aber alles allein uf die luter [lautere, i. S. v. pure] gottheit reicht. ${ }^{51}$

\subsection{Johannes Brenz' Voten auf dem Uracher Götzentag 1537}

Nachdem Brenz sich zwar anfänglich als radikaler Bilderkritiker ausgezeichnet hat und er auch in den Fragstucken von 1528 eine bilderkritische Position vertrat, erscheint es doch verwunderlich, dass aus der Haller Reformationsgeschichte kein Bericht über einen Bildersturm vorliegt. ${ }^{52}$ Lediglich bei der Festlegung der zu haltenden Feiertage scheint es schon damals zu Auseinandersetzungen zwischen dem Feierbedürfnis der Jugend und wirtschaftlichen Interessen auf der einen Seite sowie religiösen Bedürfnissen auf der anderen gekommen zu sein. ${ }^{53}$ Mit dem Beginn seines Wirkens in Württemberg wird Brenz erneut mit der Bilderfrage konfrontiert. Doch nun geht es nicht mehr „nur“ um die Frage, ob Bilder anzufertigen sind oder nicht, sondern ganz konkret, was mit den bisher geschaffenen Bildern in den Kirchen geschehen solle. Auslöser für die Frage war

$50 \mathrm{Zu}$ Leo Juds Bilderkritik vgl. Stirm: Bilderfrage (wie Anm. 6), $154 \mathrm{f}$.

51 Ludwig Hätzer (Protokoll), gesprech anbetreffend die götzen und die meß (Oktober 1523), (zitiert nach Berns [wie Anm. 49], Bd. 1, Nr. 18, S. 295).

52 In den Haller Kirchenordnungen der 1520er Jahre taucht die Bilderfrage an keiner Stelle auf. In der Frühmessordnung von 1526 geht es nur um den Ablauf und die zu haltenden Gebete (EKO XVII/1, Nr. 1, 37-41). In der Kirchenordnung von 1527 erwähnt Brenz lediglich, dass in der Alten Kirche die Taufen nur an Ostern und Pfingsten gefeiert wurden und nicht wie aktuell am Gedenktag der hailigen, wie andere feyertag, zugeaignet warn, wie in Canonibus erfunden wurt (EKO XVII/1, Nr. 2, 43). Im Blick auf potentielle Änderungen der Haller Zeremonialordnungen, zu denen auch eine mögliche Bilderentfernung gehören würde, verlangt die Kirchenordnung von 1527: Und ob etwas anders in der kirchen ausserthalben der gemeinen ordnung zuthon were, solt es vorhin an die bestimpten von der Oberkait gelangen, von den selbigen ainer gantzen Oberkait furgebracht werden, welche, so es nutzlich fur die kirchen wurd angesehen, approbirt oder, so es fur untuglich geachtet, verwurffen, darmit nit einer itliche sonderliche person irs gefallen in der kirchen ordnung zu stolzieren und leben gestat wurd (EKO XVII/1, Nr. 2, 58).

53 In der Kirchenordnung von 1527 werden als einzuhaltende kirchliche Feiertage genannt (EKO XVII/1, Nr. 2, 53 f.): alle Sonntage, die Gedenktage der Apostel, Christfest (25.12.) und Stephanustag (26.12.), Neujahr, Dreikönige, Lichtmess (2.2.), Mariae Verkündigung (25.3.), Ostern und der Tag danach, Himmelfahrt, Pfingsten mit dem folgenden Tag, Johannistag (24.6.), Heimsuchung Mariens (2.7.), Maria Magdalena (22.7.) und Allerheiligen (1.11.). Im selben Jahr predigte Johannes Brenz noch anlässlich der Einführung der neuen Feiertagsordnung: Wie wol jungstverschiner tagen ein erbar Rath uff vilfeltige furgebracht clagen, so diser Zeit unter den gewerbenden und handtwercksleutten allerley unordnung der feiertag halber erwachssen, ein verpot, an was tagen man feiren oder nyemant offentlich arbeiten oder fail haben solle (EKO XVII/1, Nr. 4c, S. 78 f.). 
ein Erlass Herzog Ulrichs (1487-1550) von 1536, wonach zuerst in Stuttgart und dann im ganzen Herzogtum alle Heiligenbilder aus den Kirchen zu entfernen waren:

Im Jahr 1536, den 8. Maii wurden die Bilder der Heiligen aus denen Kirchen zu Stuttgardt weggeschafft, und denen Closters-Personen beederley Geschlechts die Erlaubniß zu heurathen gegeben. [...] Am Montag nach Pfingsten [d.h. am 5. Juni 1536, TK] wurden auf dem Marckt zu Stuttgardt folgende Puncten ausgeruffen: 1. Niemand setze sich wieder die Evangelische Predigten aus dem Wort GOttes. [...] 3. Niemand lauffe anderstwohin Messen zu hören [...] 5. Die Bilder, welche man anbettet, sollen mit Vorwissen der Obrigkeit und des Predig-Amts weggethan, die unärgerlichen aber geduldet werden. ${ }^{54}$

In den folgenden Jahren kam die Frage auf, was mit den ,unärgerlichen aber geduldet(en)“ Bildern gemeint sei. Denn in Württemberg waren sowohl Vertreter der oberdeutschen bzw. Schweizer Reformation als auch Vertreter der mitteldeutschen Reformation führend tätig. Während für Zwingli und die meisten oberdeutschen Reformatoren die Bilderfrage einen status confessionis hatte, wandte man sich in Wittenberg nach den Unruhen des Winters 1521/22 ${ }^{55}$ entschieden gegen jede Form des Bildersturms, denn die bild und altarien in den kirchen soellten auch abgethon werden, damit abgoetterey zu vermeyden, dann drey altaria on bild genug seind. ${ }^{56}$

Johannes Brenz zählte in Württemberg mit Erhard Schnepf (1495-1558) zu den Vertretern der lutherischen Reformation. Ambrosius Blarer (1492-1564) dagegen war Vertreter der Zürcher bzw. oberdeutschen Positionen. ${ }^{57}$ Man traf sich am 10. September 1537 in Urach, um dort gemeinsam über das weitere Vorgehen in der Bilderfrage zu disputieren. ${ }^{58}$ Schnepf und Blarer nahmen als die beiden Visitatoren des nördlichen (,unter der Steig“) und südlichen („ob der Steig“) Her-

54 Martin Crusii: Schwäbischer Chronick. Zweyter Band, 3. Theil Eilfftes Buch 11tes Capitel, Franckfurt / Leipzig 1738, S. 241.

55 Vgl. Natalie Krentz: Ritualwandel und Deutungshoheit. Die frühe Reformation in der Residenzstadt Wittenberg (1500-1533) (Spätmittelalter, Humanismus, Reformation 74). Tübingen 2014, S. 186-214, hier insbesondere 200-210, die davon ausgeht, dass die Wittenberger Unruhen gar nicht so bedeutend waren, wie sie später dargestellt wurden, sondern durch den Kurfürsten und seine Berater genutzt wurden, um gegenüber der Stadt und damit dem Kurfürstentum die „Ausübung von Definitionsmacht in geistlichen Belangen durchzusetzen“ (S. 209).

56 Ordnung des Rates der Stadt Wittenberg vom 24.1.1522, abgedruckt in: Martin Luther Studienausgabe Bd. 2. Berlin 1982, S. 527,20 f. Damit liegt die Wittenberger Stadtordnung argumentativ genau auf Luthers Linie, der die Bilder ablehnt und deren Beseitigung fordern kann, sofern durch sie Abgötterei hervorgerufen wird. Vgl. auch Litz (wie Anm. 25), S. 24 mit Anm. 13, die sich vor allem auf die dritte Invokavitpredigt Luthers vom 11.3.1522 bezieht.

$57 \mathrm{Zu}$ Blarers bilderkritischem Wirken im Vorfeld seiner Anstellung im Herzogtum vgl. Litz (wie Anm. 25), S. 41-49.

58 Zum Uracher Götzentag grundlegend Ehmer (wie Anm. 8), S. 67-91; Litz (wie Anm. 25), S. 4956, die vor allem Blarers Positionen wiedergibt. 
zogtums teil, Brenz als Vertreter der Tübinger theologischen Fakultät. Die weiteren beteiligten Theologen waren der Herrenberger Pfarrer Kaspar Gräter (um 1501-1557), der Uracher Pfarrer Wenzeslaus Strauß († 1552/53), der Tübinger Theologieprofessor Paul Constantin Phrygio (um 1483-1543). Aus der Reichsstadt Reutlingen erschienen noch Matthäus Alber (1495-1570) und Johannes Schradin $(† 1560 / 61) .{ }^{59}$

Brenz äußerte bei der Disputation in mehreren Gesprächsgängen seine Einstellung zu den Bildern. ${ }^{60}$ Zuerst teilte Erhard Schnepf auch im Namen von Johannes Brenz jedoch sein grundsätzliches Unverständnis mit, wieso überhaupt die herzoglichen Räte und damit der Herzog über dieses Thema verhandeln, dies sei dezidiert eine theologische Fragestellung, die darum auch allein durch eine der theologischen Fakultäten in Tübingen, Wittenberg oder Marburg zu klären sei. ${ }^{61}$ Brenz selbst zeigt sich in seiner ersten Meldung erstaunt darüber, dass es in der Disputation nicht nur darum geht, welche Bilder evtl. aus den Kirchen entfernt werden sollen, sondern die Grundsatzfrage aufgeworfen wurde, ob überhaupt Bilder in den Kirchen sein dürften. Dies steht für ihn nicht im Einklang mit dem herzoglichen Erlass von 1536. Zugleich fragt er sich, welche Rolle er als Vertreter der Tübinger Fakultät in der Anhörung habe - ob er Zuhörer, Vermittler oder eine betroffene Partei sei. ${ }^{62}$ Aus dem folgenden Gesprächsverlauf wird deutlich, dass die herzoglichen Räte ihn als Partei ansehen, denn sie insistieren darauf, dass Brenz wie auch alle anderen zu der Grundsatzfrage, ob weiterhin Bilder in den Kirchen angebracht sein dürfen, Stellung nimmt. ${ }^{63}$

Brenz bringt dagegen folgende Einwände:

1. Wenn es anstößige Bilder gibt, dann mögen diese durch die Obrigkeit entfernt werden, wie durch die Obrigkeit auch ganze Kirchen bei Bedarf entfernt werden könnten.

2. Die Forderung von Ambrosius Blarer, alle Bilder aus den Kirchen abzuschaffen, sorgt nur für Zwang und Not bei den Christen. Das widerspricht aber dem Evangelium.

$59 \mathrm{Zu}$ den theologischen Teilnehmern und ihren Positionen Ehmer (wie Anm. 8), S. 68 ff. Dort auch die herzoglichen Räte, die an der Disputation mitwirkten (S. 70 f.).

60 Das ursprüngliche Protokoll des Götzentages ist nicht mehr erhalten, der Gesprächsverlauf wird aber wiedergegeben von Christian Besold: Virginum Sacrarum Monimenta In Principvm Wirtenbergicorvm Ergastulo Litterario: Justâ Annorum Centuriâ, Injustâ detenta Captivitate, Tandem Svperis Faventibvs, [...] Praeunte perbrevi Contentorum omnium Compendio, Juridicis, aliisq[ue] prout rerum varietas exigebat, stipata Notationibus, in Lucem prodeunt [...]. Tübingen 1636, S. 88-97. Nach Ehmer (wie Anm. 8), S. 66, hat wohl Christian Besold selbst das Protokoll während seines Wirkens als Stuttgarter Regierungsrat zwischen 1634-36 entwendet.

61 Besold (wie Anm. 60), S. 89. Für Ehmer (wie Anm. 8), S. 71 f., ist es eindeutig ein Schiedsgericht, was sich auch im weiteren Verlauf deutlich zeigte.

62 Besold (wie Anm. 60), S. 92.

63 Ebd., S. 93: Es haben aber die Räth die Frag zuem Überfluß noch einmahl lassen herumb gehen / Ob die Bilder mögen auß der Kirchen gethan werden. 
3. Sollte der Herzog sich dazu entscheiden, die Bilder zu entfernen, würde dies mit Sicherheit zu Verstimmungen gegenüber den anderen lutherischen Herrschern führen, da die Abschaffung der Bilder in Württemberg impliziere, dass die reformatorischen Regionen, in denen es noch Bilder gibt, im Unrecht seien.

4. Es gibt Kirchen, in denen nicht mehr der Dekalog verkündet wird. ${ }^{64}$ Würde man nun auch noch die Bilder abschaffen, würde der (positive) moralische Eindruck derselben auch verloren gehen.

5. Sollte Württemberg die Bilder abschaffen, besteht die Gefahr, überall als zwinglianische Sekte zu gelten. ${ }^{65}$

6. Wollte man alle Ärgernisse in den Kirchen beseitigen, dürften sich auch nicht mehr die jungen Leute in den Kirchen gegenseitig ansehen. ${ }^{66}$

Da es keine Einigung, noch nicht einmal eine mögliche Kompromisslinie gab, ${ }^{67}$ wurden die beteiligten Theologen aufgefordert, ihre Positionen noch einmal schriftlich niederzulegen. Dies taten Johannes Brenz, Erhard Schnepf und Wenzeslaus Strauß noch am selben Tag in einer gemeinsam an den Herzog gerichteten Bittschrift.

Nach einer grundlegenden Bejahung von Bildern in Kirchen, ${ }^{68}$ argumentieren sie nun dezidiert exegetisch, anders als in der vorherigen Anhörung. Zwar stellen sie fest, dass es im Alten Testament nicht ausdrücklich heißt „Du solt Bilder haben!“ Doch unter Verweis auf Num 15,38-41 und Dtn 6,8 f. zeigen sie, dass es dort auch schon äußere Zeichen (nämlich Quasten an den Gewändern, Gebetsriemen

64 Möglicherweise spielt Brenz hier auf Dekalogtafeln an, deren Existenz in spätmittelalterlichen Kirchen etwa in der Form von Katechismustafeln belegt ist. Vgl. Hans Jürgen Rieckenberg: Die Katechismus-Tafel des Nikolaus von Kues in der Lamberti-Kirche zu Hildesheim. In: DA 39 (1983), S. 555-581; Hartmut Boockmann: Über Schrifttafeln in spätmittelalterlichen deutschen Kirchen. In: DA 40 (1984), S. 210-224, hier 212. Boockmann vermutet, dass es sich bei diesen Tafeln wohl oft um Einblattdrucke handelte, die auf Holztafeln in den Kirchen angebracht wurden. Er verweist hierfür auf den Bericht des anonymen Biberacher Geistlichen (vgl. oben Anm. 11), $7 \mathrm{f}$.

65 Vgl. Volker Leppin: Theologischer Streit und politische Symbolik. Zu den Anfängen der württembergischen Reformation. In: Archiv für Reformationsgeschichte 90 (1999), 159-187, wieder abgedruckt in: Ders.: Reformatorische Gestaltungen. Theologie und Kirchenpolitik im Spätmittelalter und Früher Neuzeit, Leipzig 2016, S. 153-176, hier 154 f., 165 f.: „Das war angesichts des Kaadener Vertrags [von 1534, TK] keine rein konfessionelle Aussage, sondern es enthielt die Drohung, dass Ulrich mit der Bildentfernung seine rechtlichen Grundlagen tangieren würde.“ (166).

66 Besold (wie Anm. 60), S. 93 f.

67 Nach Ehmer (wie Anm. 8), S. 82 f., wäre ein möglicher Kompromiss auf der Linie von Matthäus Alber zu finden, der sich dafür aussprach, die Bilder, die zugunsten von Messen gestiftet wurden, abzuschaffen, aber im Blick auf die Schwachen sich damit Zeit zu lassen. Litz (wie Anm. 25), S. 51 f., sieht Alber theologisch viel näher an Brenz und Schnepf, als dass seine Position wirklich als Kompromiss hätte dienen können.

68 Supplication an Herzog Ulrich von Württemberg der Bilder halb von Joh. Brenz, M. Wentz und M. Erhard Schnepff (10. September 1537). In: Anecdotia Brentiana Nr. 70, S. 192-199 (im Folgenden: Supplication): die bilder, [...], sind Gottes wort nit allein onhinderlich, sonder dem selben gemaess und seiner gestalt fürderlich (S. 192); unser onergerlich bildnuss und gemaelde biblischer historien [...] zu ermahnen nutzlich (S. 193). 
und Mesusot) zum Ansehen gab, um die Menschen an Gott zu erinnern. ${ }^{69}$ Und wenn Christus in Mt 23,13-36 seine Weherufe über die Priester und Schriftgelehrten ausstößt, dann tut er das nur, weil der Juden sacrificia, opffer und denckmal zur abgotterei geradten seindt. ${ }^{70}$

Direkt wird Zwingli als Urheber der Bilderkritik angegangen. Denn dieser habe in seiner Sakramentenlehre durch die Trennung von innerem und äußerem Wort schon dafür gesorgt, dass jedes „,Denckzeichen“, ${ }^{71}$ und damit auch die von Christus eingesetzten Sakramente Taufe und Abendmahl, per se gegenüber Gottes Wort hinderlich seien und darum entfernt werden müssten. ${ }^{72}$

Doch „Denckzeichen“ finden sich auch im Neuen Testament. Paulus spricht davon, dass er nichts wissen will als allein den Gekreuzigten (vgl. 1 Kor 2,2), womit auch Kreuze und Kruzifixe in den Kirchen gemeint seien, die aber Gottes Wort nicht hinderlich seien. ${ }^{73}$ Nach einem kurzen Rekurs auf die Kirchenväter wird noch einmal Brenz' Argumentation aus der Disputation aufgenommen, wonach es gegenüber den anderen Kirchen ${ }^{74}$ unklug wäre, durch die Abschaffung der Bilder so zu tun, als ob die etwas in iren kirchen gottes wort zuwider hetten. $^{75}$

Im Übrigen sei es in den Orten, in denen Bilder entfernt wurden, immer wieder zu Aufruhr gekommen und der Bildersturm selbst habe seine Wurzeln in den „Carlstadischen und Zwinglischen“ Sekten, die durch ihre Forderungen die Freiheit in den Adiaphora völlig einschränken. ${ }^{76}$ Zuletzt appelliert man an den Herzog, er möge doch bei seinem Erlass zu Beginn der Visitation in Nürtingen bleiben und die unärgerlichen Bilder belassen, weil alles andere auch in der Bevölkerung nur neue Unruhe hervorrufen wird. Gleichzeitig bittet man darum, sich weiteren Rat diesbezüglich in Wittenberg zu holen. Das achten wir E.F.G. erlich und nutzlich, auch dem frieden der kirchen gantz dienstlich. ${ }^{77}$

Durch Ambrosius Blarer wurde aber bereits am 7. Oktober 1537 in Blaubeuren ein Erlass zur Bilderfrage herausgegeben, der deutlich macht, dass Herzog U1rich sich der Meinung Blarers angeschlossen hat. Die noch in Urach diskutierte Frage nach ärgerlichen und unärgerlichen Bildern wird fallen gelassen: Das alle

69 Ebd., S. 193.

70 Ebd.

71 Man lehnt sich damit an die von Brenz seit seinem Katechismus von 1535 verwendete Formulierung des Sakraments als göttlich Wortzeichen an. Der Begriff ,göttlich Wortzeichen“ kommt in den beiden ersten Fassungen der Fragstuck (wie Anm. 46) nicht vor, im Katechismus von 1535 lediglich in seiner Erklärung zum Nachtmahl (Abendmahl). In der Erklärung zur Taufe spricht Brenz von eyn Gotlich zeychen.

72 Supplication (wie Anm. 68), S. 194.

73 Ebd.

74 Gemeint sind damit die Kirchen, die die Confessio Augustana von 1530 als gemeinsamen Bekenntnistext angenommen haben, d. h. alle lutherischen Kirchen.

75 Supplication (wie Anm. 68), S. 195.

76 Ebd.

77 Ebd., S. 196. 
bilder und gemäld in den kirchen uff nachfolgend mainung, form und maaß abgethon werden sollen. ${ }^{78}$

Scheinbar hat sich die radikale Züricher Auslegung durchgesetzt, ${ }^{79}$ doch spätestens mit der Einweihung der neuen Stuttgarter Schlosskirche im Jahre 1562 und den dort eingebrachten Steintafeln von Sem Schlör zum Glaubensbekenntnis am Altar, den Evangelistensymbolen an Kanzel und Deckengewölbe (zusätzlich wird hier noch Simson dargestellt) und einer Darstellung der Verklärung Christi ebenfalls an der Kanzel ${ }^{80}$ kehrt das Herzogtum grundsätzlich zu einer bilderbejahenden Position zurück, auch wenn in den südlichen Landesteilen („ob der Steig“") weiterhin der reformierte Einfluss vorhanden blieb.

\subsection{Das Bilderverbot im Exoduskommentar von 1539}

Anders als von Ambrosius Blarer ${ }^{81}$ ist nicht bekannt, wie Johannes Brenz auf die Entscheidung des Herzogs reagierte. Im April 1538 verließ er die Universität Tübingen und kehrte nach Hall zurück. ${ }^{82}$ Noch im selben Jahr ${ }^{83}$ arbeitete er an einem lateinischen Kommentar zum Buch Exodus, der 1539 veröffentlicht wur-

78 Rainer Henrich: Das württembergische Bilderdekret vom 7. Oktober 1537 - ein unbekanntes Werk von Ambrosius Blarer. In: BWKG 97 (1997), S. 9-21, hier 17; Litz (wie Anm. 25), S. 55 f.

79 Für Leppin (wie Anm. 65), S. 166-171, übernimmt der herzogliche Erlass von 1537 und in der Bestätigung von 1540 zwar die theologische Argumentation von Ambrosius Blarer, auch wenn dieser 1538 des Landes verwiesen wurde. Doch wichtiger noch sei für Herzog Ulrich gewesen, durch die Beseitigung aller Bilder eindeutig klarzustellen, dass man evangelisch sei. Denn eine „Belassung der Bilder verwischt offenkundig die Grenzen zum altgläubigen Kirchensystem, lässt gegenüber der radikalen Bilderentfernung Eindeutigkeit vermissen“ (S. 169). Die Württemberger Landeskinder hatten, so Leppin, durch die umliegenden oberdeutsch geprägten Reichsstädte ja schon eine Vorstellung gewonnen, wie es in einer evangelischen Kirche auszusehen habe. „Die prinzipielle Streitfrage des Uracher Götzentages nach dem Wert der Bilder umging und unterlief Herzog Ulrich. Nicht sie war es, die ihn interessierte, sondern ihn interessierte offenbar, wie die Neuheit der reformatorischen Lehre in einer oberdeutsch geprägten Gegend am besten zum Ausdruck komme: und das konnte nur durch die Bilderentfernung geschehen oder eben durch wirklich neue Bilder“" (S. 171). Vgl. jetzt auch Ders.: Habsburg vor der Tür. Zu den Bedingungen der württembergischen Reformation von Herzog Ulrichs Vertreibung bis zum Interim. In: Kirche und Politik am Oberrhein. Reformation und Macht im Südwesten des Reiches. Hg. von Ulrich A. Wien/Volker Leppin (Spätmittelalter, Humanismus, Reformation 89). Tübingen 2015, S. 71-95, hier 93: „Wer die Grenzen zum Territorium Württembergs überschritt, konnte in den Kirchenausstattungen wie im Gottesdienst auffällig wahrnehmen, dass er hier protestantischen Boden betreten hatte."

80 Vgl. hierzu die unveröffentlichte Magisterarbeit von Günter Memmert: Die Schlosskirche im Alten Schloss zu Stuttgart. Ein protestantischer Kirchenraum der Renaissance im Herzogtum Württemberg. Magisterarbeit am Institut für Kunstgeschichte der Universität Stuttgart, masch. 1999 (ein Teildigitalisat unter: http://www.kirchen-online.com/content/k_k-in-stuttgart/schlosskirche.html [abgerufen am 17.2.2017]), S. 70-91; Leppin (wie Anm. 65), S. $171 \mathrm{f}$.

81 Vgl. Henrich (wie Anm. 78), S. 9 ff.

82 Vgl. hierzu den Brief von Joachim Camerarius an Philipp Melanchthon vom 9.4.1538 (Melanchthons Briefwechsel. Texte Bd. 8. Bearb. von Christine Mundhenk. Stuttgart-Bad Cannstatt 2007, Nr. 2018).

83 Vgl. hierzu seinen Brief an den württembergischen Kanzler Nikolaus Meyer vom 17.7.1538, abgedruckt als Vorwort im Kommentar. 
de, von dem wiederum sein Drucker, Sebastian Coccius, 1540 eine deutsche Übersetzung herausgab.

Brenz setzt sich in seiner Kommentierung des Dekalogs im 20. Kapitel mit dem Bilderverbot auseinander. ${ }^{84}$ Während es in Luthers Exodus-Kommentar keine Erläuterung zur Zählung der Gebote gibt, verweist Brenz zu Beginn seiner Ausführungen darauf, dass manche das Bilderverbot zum ersten Gebot zählen, andere dagegen die Gebote voneinander getrennt zählen. Er habe sich aber dafür entschieden, der allgemeinen Unterteilung (vulgatam divisionem) zu folgen, das Bilderverbot mit zum ersten Gebot zu fassen. ${ }^{85}$

Für seine folgende Auslegung des ersten Gebots wird Brenz' hermeneutische Entscheidung zentral, die Gebotstafeln nicht als Texte zu verstehen, die entweder etwas verbieten oder etwas gebieten. ${ }^{86}$ Denn in jedem der zehn Gebote ist zugleich Ge- und Verbot enthalten. Und so legt er zuerst in sechs überaus knappen Punkten dar, welche zu bejahenden Glaubensaussagen im ersten Gebot enthalten sind, nämlich: 1) an Gott zu glauben, 2) seine Wesenseinheit (essentia unus) zu erkennen, 3) dass es derselbe Gott ist, der Israel aus Ägypten führte und die Patriarchen schon auf Christus hinwies (promissit Patriarchis Christum), 4) an den richtenden Gott für die Ungläubigen, aber den barmherzigen für die Glaubenden, 5) allein an ihn zu glauben, 6) ihm von ganzem Herzen, mit aller Macht und mit ganzer Seele zu vertrauen (vgl. Dtn 6,4f.). ${ }^{87}$

Wesentlich umfangreicher fällt dagegen Brenz' Darlegung der Verbote innerhalb des ersten Gebots aus.

Neben dem Verbot, an fremde Götter zu glauben, fasst er zugleich darunter das Vertrauen auf Heilige, egal ob lebende oder bereits verstorbene (in homines sanctos, seu vivos seu mortuos), so wie man sich überhaupt auf keinen Menschen oder auf menschliche Gerechtigkeit verlassen sollte. Brenz nimmt hier immer wieder Bezug auf Paulus und seine Gesetzesdeutung im Römerbrief (vgl. Röm 7,7-25). ${ }^{88} \mathrm{Zu}$ den Verboten gehört auch das Bilderverbot, welches aber allein der mosaischen Bürgerschaft geboten ist. ${ }^{89}$ Denn bei dem Bilderverbot handelt es sich lediglich um ein äußeres Gebot für die Gottesdienstgestaltung innerhalb des

84 In Exodum Mosi Commentarius, autore Ioanne Brentio, Halae Suevorum in officinia Petri Brubachij Anno M.D. XXXIX, cum gratia \& privilegio Caesareo ad quinquennium (Köhler 102), (im Folgenden Comm. in Ex.) Cap. XX. (S. 180a-182b). Die deutsche Übersetzung: Das ander Buch Mosi Exodus genant vormals durch H. Johansen Brentzen in lateinischer Sprach außgelegt jetzund vertewtscht durch Sebastian Coccium. Getruckt zu Hagenaw 1540 (Köhler 109).

85 Ebd., S. 180a. Zu den verschiedenen Fassungen des Dekalogs bei Brenz und deren Herkommen vgl. Weismann (wie Anm. 46), S. 138-144. Demnach zitierte Brenz den Text des Bilderverbots in keinem seiner Katechismen.

86 Comm in Ex. (wie Anm. 84): quidam dividunt praecepta in Negativa et Affirmativa (S. 180a).

87 Ebd., S. 180a-180b.

88 Ebd., S. 180 b.

89 Ebd., Sequuntur nunc quaedam personalia seu caeremonialia, quae tantum ad politeian Mosaicam pertinuerunt (S. 181a). Vgl. dazu auch Martin Luthers Deutung des Textes allein auf die Juden (oben Anm. 19). 
Judentums..$^{90}$ Er verweist in seiner Argumentation auf den sich unmittelbar an den Dekalog anschließenden Text von Ex 20,22-26, in dem es neben der alleinigen Verehrung Gottes und dem Bilderverbot auch um Brand- und Tieropfer geht, und Dtn 5,32, wonach die Kultbildbestimmungen für Israel absolut gesetzt sind. So symbolisiert für ihn auch der Tanz der Israeliten um das goldene Kalb (Ex 32,1-6) den damaligen Irrglauben, einen rechten Gottesdienst zu feiern für den Gott, der die Israeliten aus Ägypten geführt habe. ${ }^{91}$ Deswegen gilt das Bilderverbot auch nur den Israeliten, für die Christen hat es keine Bedeutung, weil diese in ihren Gottesdienstformen von Gott keine allseits gültige Regelung aufgetragen bekamen. ${ }^{92}$ Als weiteren Beleg, dass für die Christen das jüdische Gesetz nicht mehr gilt, verweist er auf das nicht mehr geltende Verbot, Schweinefleisch zu essen und auf die von Christen nicht mehr praktizierte Beschneidung: „Nachdem aber nun Christus gekommen ist, sind solche mosaischen Gebräuche aufgehoben, und jeder ist für sich frei darin, Bilder von Christus oder den Heiligen zu besitzen, solange er es nicht gottlos missbrauche. ${ }^{\text {(993 }}$

\subsection{Das Bilderverbot im Catechismus explicatus von 1551}

Nach dem Schmalkaldischen Krieg musste Johannes Brenz Hall verlassen, da seine Sicherheit vor Ort nicht mehr gewährleistet werden konnte. Er trat nun dauerhaft in den württembergischen Dienst. Da nach dem Augsburger Interim alle evangelischen Pfarrer ihre Ämter verloren, nutzten die württembergischen Herzöge eine Lücke des Interims, um diese Geistlichen weiterhin anzustellen nun aber als Katechismuslehrer, die so die geistliche Versorgung der Evangelischen sicherstellen sollten. In dieser Situation verfasste Brenz einen umfangreichen Kommentar zu seinen Fragstucken von 1535, der auf noch in Hall gehaltenen Predigten beruhte. 1551 erschien der Katechismus in lateinischer Sprache, ab 1552 in deutscher Fassung, übersetzt durch Hartmann Beyer. ${ }^{94}$ In seinem großen Katechismus gibt Brenz im Wesentlichen seine Argumentation aus dem Exoduskommentar wieder. Das Bilderverbot wird auch hier im Rahmen der Auslegung zum Dekalog behandelt. Aufgrund des Lehrbuchstils systematisiert Brenz aber bestimmte Fragestellungen noch stärker.

So erklärt er gleich im ersten Teil, ob denn das alttestamentliche Gesetz im Neuen Testament noch gültig sei. Innerhalb des Dekalogs gäbe es nämlich zwei Ge-

90 Comm in Ex. (wie Anm. 84), S. 181a: primum ne usurpent alia sacra, aliosque sacrificandi et colendi Deum ritus.

91 Ebd.: ista sunt vere divina sacra [...] et propter quae meremur gratiam Dei, qui nos eduxit de Aegypto.

92 Ebd., S. 181a/b: nec obligat Christianos, quibus non est aliqua certa forma publicorum sacrorum, aut ecclesiasticarum ordinationum divinitus praescripta.

93 Ebd.: Postquam autem Christum advenit, caeremonica Mosaica sublata est, et per se liberum est, simulacra Christi ac sanctorum publice habere, tantum ne quis impie ipsis abutatur.

94 Vgl. zur Entstehung des Catechismus explicatus Weismann (wie Anm. 45), S. 131. 
bote, die sich allein auf den jüdischen Kultus bezögen und darum für Christen nicht mehr gelten. Zum einen ist dies das Bilderverbot, zum anderen das Sabbatgebot. ${ }^{95}$ Und darum können Christen auch Bilder in ihren Kirchen haben, insofern diese nicht angebetet werden.

Mit demselben Gedanken beginnt Brenz seine ausführliche Auseinandersetzung mit dem Bilderverbot. Dieses hat zwei Ebenen - zum einen als rein jüdisches Zeremonialgebot, zum anderen als ein für alle gültiges Moralgesetz. ${ }^{96}$

Auf der Ebene des jüdischen Zeremonialgesetzes bildet es seinen Bezug zu anderen Kultvorschriften des Alten Testaments, z. B. dem Verbot, Schweinefleisch $\mathrm{zu}$ essen oder Mischgewebe zu tragen. Dabei finden sich auch im Alten Testament schon immer Bilder. Brenz verweist dafür etwa auf Cherubendarstellungen (Ex 25,18-22 u. ö.). Wie in der modernen Religionssoziologie deutet Brenz darum das Bilderverbot als Reaktion des Volkes Israel, um nicht den umliegenden Völkern zu gleichen, auch wenn sie punktuell doch Bilder nutzten.

Im Blick auf das Bilderverbot gilt für Christen: „Auch wenn es aus vielerlei Gründen besser wäre, wenn man keine Bilder öffentlich in unsere Kirchen stellte, ist es dennoch an sich keine Sünde, sondern man ist frei, Bilder zu besitzen, besonders wenn sie zur Erinnerung an frühere Taten dienen. ${ }^{\text {(997 }}$

Gleichzeitig ist es aber auch so, dass allen Menschen eine natürliche Gotteserkenntnis gegeben ist. ${ }^{98}$ Darum können alle Menschen erkennen, dass jede Form von Bilderverehrung die rechte Gottesverehrung zerstört. Dies gilt Juden und Christen gleichermaßen und entspricht so dem ersten Gebot. Sobald also Bildern Verehrung oder gar Anbetung zukommt, verletzt dies das erste Gebot. Und so kann Brenz resümierend schreiben: „Deshalb ist es auch den Christen nicht verboten, Bilder von Christus und den Heiligen als Erinnerung an frühere Taten $\mathrm{zu}$ besitzen, aber es ist verboten, dass sie die Bilder verehren oder sie anbeten. ${ }^{* 99}$

95 Catechismus pia et utili explicatione illustratus Ioanne Brentio autore, I. Corint 14, Ne sitis pueri sensibus, Sed malicia pueri sitis, sensibus vero perfecti M.D.LI (Köhler 197) (im Folgenden : Catech. expl.): Ac duae quidem tantum Caeremoniae recitantur in decalogo. Una est de statuis et de simulacris, seu sculptilibus, in primo praecepto. Altera est de observatione diei Sabbati, in tertio praeceptio. (S. 409). Die deutsche Übersetzung: Heilsame und nuetzliche Erklaerung des Ehrwidrigen Herren Joannis Brentij uber den Catechismus Durch Hartman Beyer allen Christlichen Hausvaettern zugefallen, verdertscht. 1. Corinth. 14 [...]. M.D.LII. (Köhler 211).

96 Ebd., S. 457: alterum est Ceremoniale, seu Leviticum, seu Iudaicum. Alterum est Naturale, seu Morale.

97 Ebd., S. 459: Quanquam enim utilis esset, propter multas causas, ut nullae imagines, publice in templis nostris proiterant, tamen per se non est peccatum, sed liberum habere imagines, praesertim in rerum gestarum monumentum. Mit seinem Verweis auf die res gestae bewegt sich Brenz nun wiederum ganz auf der Ebene, wie er sie zu Beginn seiner Beschäftigung mit der Bilderfrage noch explizit abgelehnt hat, der Position, wie sie von Gregor dem Großen vertreten wurde (vgl. oben Anm. 32).

98 Ebd., S. 459: et prohibitur non scripta tantum lege, sed etiam naturali lege, quae est omnibus hominibus insculpta.

99 Ebd., S. 459 f.: Itaque etsi Christianis non est prohibitum, habere imagines Christi et Sanc- 


\section{Zusammenfassung}

Martin Luther befasst sich mit dem Bilderverbot schon in seiner Predigttätigkeit vor seinem Wirken als Reformator. Er ordnet dabei das Bilderverbot dem ersten Gebot unter und sieht in diesem lediglich eine Konkretisierung des Verbots, anderen Göttern zu dienen. Für ihn ist das Bilderverbot darum auch kein äußerliches Erkennungszeichen, das unmittelbar den Christen gilt, und verlangt mithin durch die christliche Gemeinde auch keine besondere Beachtung. Strukturell sind Bildersturm und Bilderverehrung für ihn nur zwei Seiten einer Medaille und werden zum Ärgernis, wenn beides die wahre Gottesverehrung verhindert, nämlich auf Christus zu schauen.

Das Bilderverbot begleitete Johannes Brenz über fast drei Jahrzehnte seines Wirkens. Dabei lässt sich in dieser Zeit ein völliger Wandel seiner Positionen feststellen. Als junger Haller Prädikant und Reformator vertritt er 1524 radikal bilderkritische Positionen. Gottes- und Heiligenbilder dürfen nicht angefertigt werden. Er lehnt auch explizit den pädagogischen Charakter der Kultbilder ab. In seiner Auslegung zu Joh 1,18 orientiert er sich dann 1527 und 1528 an einem traditionell ikonoklastischen Argument, wonach die göttliche Natur Christi nicht darstellbar sei. Aufgrund der fehlenden Berichte über einen möglichen Bildersturm in Hall lässt sich aber schließen, dass Brenz nie versuchte, seine Forderungen aus dieser Frühphase in die kirchliche Praxis der Reichsstadt umzusetzen. Erst mit dem Beginn seiner Wirksamkeit in Württemberg stellt sich für Brenz die Frage, ob er selbst aktiv an der Beseitigung der Kultbilder mitwirken möchte. Was genau bei ihm zur Änderung seiner Position geführt hat, können wir heute nicht mehr feststellen. Aber zwischen 1532, d. h. der zweiten Auflage der Fragstuck von 1528, und 1537 nimmt er eine neue Position ein. Im Anschluss an Herzog Ulrichs Erlass, dass die ärgerlichen Bilder aus den Kirchen zu entfernen seien, die unärgerlichen aber weiterhin bleiben dürfen, argumentiert Brenz bei der Uracher Disputation vor allem von der Kircheneinheit her. Brenz will so verhindern, dass das Herzogtum sich aus dem Konsens der lutherischen Kirchen herausbegibt. In der sich an die Uracher Disputation anschließenden Supplication an den Herzog nimmt Brenz diesen Gedanken von der lutherischen Kirchengemeinschaft sowie der Angst vor möglichen Unruhen erneut auf, erweitert ihn aber auf die Bibelexegese, wonach es auch im Alten Testament schon immer äußere Zeichen des Glaubens gegeben habe und Paulus im Neuen Testament davon schrieb, nichts wissen zu wollen als den Gekreuzigten, womit auch Kreuze und Darstellungen desselben gemeint seien.

Im Jahre 1539 vertritt Johannes Brenz in seinem Exoduskommentar eine überaus bilderfreundliche Position. Das Bilderverbot gilt demnach nur dem jüdischen Volk, weil es ein Teil der alttestamentlichen Kultordnungen ist und im 
Neuen Testament keine Fortsetzung fand. Als Beleg dafür zieht er etwa das Verbot von Schweinefleischverzehr heran, welches für die Christen bekanntlich auch keine Rolle mehr spielt. Das Bilderverbot selbst ist ein Teil des ersten Gebots, weil Gott allein zu verehren ist und so jeder Bilderkult als Übertretung des ersten Gebots zählt.

Diese bilderfreundliche Sicht behält er dann auch in seinem Kommentar zum großen Katechismus 1551 bei. Im Rahmen der jüdischen Kultordnungen gilt das Bilderverbot für die Christen nicht mehr. Vielmehr können die Bilder durch die ihnen innewohnende Erinnerungsfunktion den Glauben stärken. Im Blick auf das erste Gebot behält es aber seine Wirksamkeit bei, denn als Menschen sind wir in der Lage, Gott zu erkennen und denn nur ihm allein gehört alle Anbetung und Verehrung. 